\title{
A study of vacuum arc ion velocities using a linear set of probes
}

\author{
Stefan Hohenbild ${ }^{1}$, Christoph Grübel ${ }^{1}$, Georgy Yu. Yushkov² ${ }^{2}$ Efim M. Oks² ${ }^{2}$ and André \\ Anders $^{3 *}$ \\ ${ }^{1}$ Universität der Bunderwehr München, Fakultät für Elektroechnik und Informationstechnik, EIT-1a, \\ Werner Heisenbergweg 39, D-85577 Neubiberg, Germany \\ ${ }^{2}$ High Current Electronics Institute, Russian Academy of Sciences, 4 Akademichesky Ave., Tomsk, 634055, \\ Russia \\ ${ }^{3}$ Lawrence Berkeley National Laboratory, 1 Cyclotron Road, Berkeley, California 94720, USA
}

\begin{abstract}
The most likely velocity of ions moving away from vacuum arc cathode spots was measured using a set of probes along the path of plasma expansion. The goal was to determine how much, if any, change of the ion drift velocity occurs in the expanded plasma. The arc discharge current was perturbed to create plasma density markers whose travel is picked up by the set of probes. It was found that the perturbation with current oscillations did not result in consistent data because ion current maxima and minima are not only determined by the plasma production but by the transients of the arc pulse and by the asymmetry of the ion velocity distribution function. Perturbation with a short current spike was more conclusive. The ion velocity was measured to be slightly reduced with increasing distance from the cathode, which can be explained by collisions of ions with the background of neutrals. The ion velocity was increased when the arc current was increased, which correlated with enhanced arc voltage and power dissipation. The ion velocity could be enhanced when the plasma was produced in a non-uniform magnetic field.
\end{abstract}

(Figures in this article are in colour only in the electronic version)

\footnotetext{
*Author to whom any correspondence should be addressed, electronic mail: aanders@lbl.gov
} 


\section{Introduction}

Numerous studies of the ion velocities of cathodic vacuum arcs have shown that the ions drift with supersonic velocities from their "birthplace,” the cathode spot, into the ambient vacuum or low pressure gas region [1-10]. It is well established that several forces contribute to ion acceleration, most importantly the ion pressure gradient and electron-ion “friction," and electrons, in turn, were accelerated by the electron pressure gradient [11-13]. Ions of different charge states flow with about the same velocity in the plasma, which is an indication that the main acceleration did not occur by the electric field of a hypothetical potential hump. However, one should realize that the approximately same ion drift velocities in the plasma imply that ions of different charge states arrive at a substrate or detector with different energies, unless those substrates or detectors were exactly at the plasma potential. This is because they are accelerated at the substrate’s or detector's boundary sheath and gain kinetic energy according to

$$
\Delta E_{i}=Q e \Delta V
$$

where $Q$ is the charge state number, $e$ is the elementary charge, and $\Delta V$ is the sheath voltage.

Various aspects of ion acceleration and ion velocities have been investigated, such as the type of material $[3,6,8]$, the current amplitude, the effect of background pressure [4, 7], and the presence of magnetic fields [6]. In practically all of the studies, a specific configuration of electrodes and measuring system was considered, and the assumption was made, more or less explicitly, that the ion acceleration occurs only in the vicinity of the cathode spots and that the drift velocity remains constant thereafter, unless collision with background gas changes the velocity distribution function towards lower values [4, 7]. In practically all experiments, a (one!) detector was places at a certain distance from the plasma-producing cathode, with a characteristic cathode-detector distance of the order $0.1 \mathrm{~m}$.

Surprisingly, there is a lack of data considering possible changes of the flow velocity (or corresponding kinetic energy) as the plasma expands from the cathode region. It is expected that those changes are small unless forces act on the ions, which is especially possible and relevant should there be a gradient of a magnetic field [6]. In this contribution, we investigate the flow of plasma on a longer scale $(\sim$ $0.5 \mathrm{~m}$ ) with a set of equally spaced ion detectors, and we consider the cases with and without magnetic field in the vicinity of the arc plasma source. 


\section{Measuring principle and experimental setup}

A convenient approach to measuring the most likely ion velocity is to use a time-of-flight method. The plasma-producing arc current can intentionally be perturbed to create "markers," and one follows how the density perturbation is streaming with the plasma [14]. Three types of perturbations have been investigated in the past, using a single ion detector, namely: (i) current oscillations [5], (ii) a sharp, single current spike [6, 15], and (iii) the abrupt termination of the discharge [8].

Each of those methods has advantages and disadvantages. The current oscillations allow for several measurements of the most likely velocity with time resolution, which is interesting for pulsed arcs where the plasma properties evolve towards steady-steady state [5]. The sharp spike perturbation is the easiest to observe and interpret but gives only one data point per measurement. Additionally, one has to be careful because the spike itself may change the plasma conditions such as the ion charge state distribution, especially when the spike amplitude is large compared to the typical arc current [15]. Time resolution is possible by varying the delay of spike relative to the ignition time of the arc discharge [6]. The abrupt termination of the discharge allows us to obtain a full distribution function, not just the most likely velocity, however, the error can be large at the high energy end of the distribution function due to the fluctuations of the density [8].

Two types of perturbations have been investigated in the present investigation: A) current oscillations and B) single spike perturbation. In contrast to previous measurements, we follow the propagation of the perturbation using several ion collectors differently spaced from the cathode, therefore looking at the velocity evolution as the plasma streams from the cathode region. Fig. 1 shows the experimental setup. The distances of the probes from the cathode surface were $6 \mathrm{~cm}, 15 \mathrm{~cm}, 25 \mathrm{~cm}, 35 \mathrm{~cm}$, $45 \mathrm{~cm}$, and $55 \mathrm{~cm}$. Each probe was negatively biased ( $50 \mathrm{~V}$ ) relative to the grounded anode via a capacitor. In this way, the collectors operated in the ion saturation regime. The signals were recorded using two, synchronically triggered, 4-channel digital oscilloscopes. The probe area was $2 \mathrm{~cm}^{2}$ each, which the probe sheath estimated to be about $1 \mathrm{~mm}$ or even less for the probes close to the source. The probes were positioned off axis so as to not shadow the flow and to minimize the effect on the flow, which could influence the probes signals downstream. The experiments with oscillating arc current give several 
velocity data points by considering the time difference $\Delta t$ between corresponding arc and ion current maxima and minima (Fig. 2). The velocity is simply calculated by

$$
\bar{v}=\frac{\Delta s}{\Delta t}
$$

where $\Delta s$ is the distance between arc cathode and probe. Additionally one can use the $\Delta s$ and $\Delta t$ values of neighboring probes to determine a most likely ion velocity that is averaged between neighboring probes, as opposed to averaged over the whole travel distance from the cathode to the probe.

The arc spots burned on the front face of a cathode rod of $1 / 4$ inch $(6.25 \mathrm{~mm})$ diameter. The rod could be exchanged to produce plasma of a different material; $\mathrm{Pt}, \mathrm{Al}, \mathrm{Cu}, \mathrm{W}$, Ti cathodes were used. The arc pulse duration was $250 \mu$ s with a pulse repetition rate of 2 pulses per second. The arc current was measured using a broad band current transformer $(0.01 \mathrm{~A} / \mathrm{V}$, Pearson) and also recorded by the digital oscilloscopes. Fig. 2 shows the arc current when an LC branch was added to produce the oscillating feature, and Fig. 3 displays the current shape with a 70 A, $10 \mu$ s (FWHM) spike superimposed onto the arc discharge by triggering a thyristor for the $1.5 \mu \mathrm{F}$ capacitor discharge via a $2 \Omega$ series resistor.

A magnetic field coil is wound around the tubular aluminum anode (inner diameter $1.8 \mathrm{~cm}$, length $3.5 \mathrm{~cm}$ ) as to produce an axial, though non-uniform magnetic field as indicated in Fig. 1. The arc discharge and magnetic field were powered independently, each having its own pulse forming network (PFN). The arc current and coil currents can be selected by the charging voltage of the corresponding PFN. The magnetic field pulse had a duration of about $600 \mu$ s and was triggered well in advance of the arc pulse, therefore, the magnetic field can be considered constant during the arc pulse. The field was calibrated using an inductive pickup sensor. The direction of the magnetic field could be reversed simply by reversing the coil current.

The experiments were done at the base pressure of the cryogenically pumped, oil-free system, which was about $10^{-4} \mathrm{~Pa}$.

\section{Results and preliminary assessment}

\subsection{Experiments with oscillating arc current}

Figure 2 shows an example of oscillating arc current and resulting probe current. The ion current signals were generally noisy and therefore we use the averaging acquisition mode of the oscilloscope to 
obtain a smooth curve for which well defined maxima and minima exist. Sometimes even averaging is insufficient to obtain ion current curves that allow us to reliably determine minimum and maximum values. In those instances, the data were discarded and the conclusions were limited to the more reliable spike method.

From the large amount of data we selected here the most revealing, illustrating the general trends. Fig. 4 shows the case of tungsten plasma in the absence of a magnetic field. One can see several features. There is a general trend that the velocity slightly decreases as time elapses. It is known from previous measurements that the plasma changes on the timescale of a few hundred microseconds or even longer. This feature has been attributed to the evolution of the neutral density in the volume into which the plasma expands [16]. The slight temporal changes occur throughout the volume in which the ion collectors are placed. Figures 5 and 6 show the same measurements but done for Pt and Al, respectively. Also here, there is a slight tendency for slowing after arc ignition. However, the different probes indicate noticeable changes of the velocity as the plasma expands: the more remote probes indicate a higher velocity, suggesting acceleration far from the spot. Even as the velocity changes are "only" 20-25\%, the associated kinetic energy is $\sim v^{2}$ and hence changes by as much as about $50 \%$.

When the magnetic field is switched on, one can observe changes in the velocity evolution (Fig.7): the velocity increases as the plasma expands away from the high field region. This should be expected from the well-known the mirror effect [17] which arises from the conservation of the magnetic moment of magnetized charged particles: the longitudinal velocity reduces when the particle moves into a region of stronger magnetic field, and reversely, the longitudinal velocity increases when the particle leaves the region of high field strength. However, one should keep in mind that the field is not strong enough that ions are magnetized. Therefore, the effect works only for electrons, and ion acceleration can only occur via electron-ion interaction.

A closer look at Fig. 7 reveals that acceleration does not occur where the greatest field gradient is located, namely close to the field coil, but rather spreads out throughout the volume, and — confusingly— one can even see a reverse trend in some of the data. Separating the data in groups obtained using the maxima (Fig. 7a-c) and minima (Fig. 7d-f) shows that the results are not consistent: there seems to be a systematic error, which is especially pronounced for the first $100 \mu$ s, i.e. the first values after triggering. 
Additionally, a major source of concern is the increased "noise" of the ion current when a magnetic field is applied. In fact, when increasing the magnetic induction to even higher values, up to the maximum of 300 $\mathrm{mT}$ possible with our setup, the fluctuation of the collector current became so large and irregular that it was impossible to determine maxima and minima which could be clearly associated with the maxima and minima of the arc current.

Therefore, we move on to the alternative principle of a single current spike, which gives a much more defined perturbation to follow.

\subsection{Experiments with a spike in the arc current}

From previous experiments [15] it is known that a sharp spike on the arc current represents a transient increase of the power dissipated, and hence one should expect that this method would temporarily shift ion charge states and velocities to higher values. Yet, the spike approach seems to be an excellent method to detect the propagation velocity of a density fluctuation in the plasma but one should keep on mind that the thus-determined values are upper values of the most likely velocities of the unperturbed plasma. Fig. 8 shows the spike in arc voltage and current used in the experiment.

By tuning the spike to different delay time and considering the differential arriving times of the ion signals at the different detectors, the spatial and temporal velocity evolution was derived (Fig. 8). In this analysis, the high frequency components of the ion current noise was removed by fast Fourier transform (FFT) using ORIGIN ${ }^{\circledR}$ software. From Fig. 8 one can see that the velocities decrease after arc initiation, as expected, but one can also see that the velocities are not constant but decrease as the plasma moves away from its "birthplace." This decrease with distance is significant and contradicts what has been found in the previous measurement using the oscillation method. Clearly, the situation requires a deeper analysis of systematic errors (section 4).

The velocity of ions may depend on the discharge conditions which can be readily varied by changing the arc current (via the charging voltage of the PFN). Fig. 9 shows that velocity increases with discharge current. The arc voltage increases about linearly with the current, and therefore the power of the discharge increases about quadratically with current. 
Finally we can also vary the magnetic field strength by the field coil. As expected, we see a clear increase with increasing field (Fig. 10). The arc current in this example is kept constant yet the arc voltage increases about quadratically with increasing magnetic field.

\section{Discussion}

The here investigated parameter space related to the velocity evolution is rather large: besides the central variables of interest, time and distance from the cathode, the parameters include cathode material, arc current, and external magnetic field. Two methods of measurements were employed, both are based on measuring how the perturbation of plasma production propagates with the plasma.

As already indicated, when looking at the data of Fig. 7a-c versus 7d-f, the measuring principle using current oscillations appears to have a systematic error. Clearly, as long at the discharge current is not constant, the amount of plasma produced cannot be assumed to be constant. Therefore the maxima or minima of ion current at the very beginning and end of the discharge should not be used. Additionally, the method implicitly assumes that the velocity distribution function does not change over time. Furthermore, minima- and maxima-derived values for the most likely velocity are only equal if the asymmetry of the ion velocity distribution function is small. Conversely, a systematic deviation between those minima- and maxima-derived values can be used to infer on the asymmetry of the distribution. We did not attempt to do this based on the noise of the ion current data. It seems more appropriate to accept the results as approximate values and put greater confidence on the spike-generated most likely velocities.

The spike method indicates that the most likely velocities slightly decrease far from the cathode spot. Given the still high plasma density in the region $\sim 0.1 \mathrm{~m}$ from the spot, there are frequent collisions in the plasma that could transfer longitudinal velocity to transverse velocity, where transverse is meant in the sense perpendicular to the direction of main plasma flow. To reconciled this transfer of kinetic energy with momentum conservation, one needs to assume that a particle component is present which is slower that the center-of-mass velocity of the plasma ions. Such slow component has been identified as neutrals, and especially metal neutrals, which fill the volume far from the cathode spot [16]. Detailed investigations of the evolution of charge states where consistent with the evolution of metal neutrals, where the source of neutrals is the plasma itself (via non-sticking ions, which "bounce back" from a surface as neutrals) and 
evaporation from macroparticles and hot cathode craters [18]. The presence of such neutrals should also cause a slowing of the plasma stream, both in time as the density of neutrals evolves, and with increasing distance, as the likelihood of a collision between a fast ion and background neutral increases. Both tendencies appear in Fig. 8.

Ion acceleration near the cathode spot is mainly caused by the electron and ion pressure gradients and electron-ion coupling. As the current is increased, the voltage is increased, too, but perhaps slightly less than indicated in Fig. 9 because no correction was made for the voltage drops at the connectors and Ohmic losses inside the cathode and anode. Therefore, we can only state in a qualitative manner that the local power density in the cathode spot region is increased when the arc current increases, which can explain the tendencies for slightly higher velocities.

Finally, looking at the data when a magnetic field is present in the cathode region (Fig. 10), we encounter a rising voltage drop across anode and cathode, which is due to the magnetic insulation of the anode. The plasma does not have a single plasma potential but a voltage drop occurs in the magnetized plasma, and electrons must cross magnetic field lines to reach the anode. Higher power dissipation and acceleration in the field gradient lead to increased ion velocities. Fig. 10 indicates, consistent with this picture, that there is no significant gain in velocity at large distances from the cathode: the velocities detected with the probes at $35 \mathrm{~cm}$ and $55 \mathrm{~cm}$ are practically the same.

\section{Conclusions}

In summary, we come to the following conclusions.

1. The two current perturbation methods employed, namely using current oscillations or a brief current spike, do not always give the same or consistent results. The culprit for the discrepancies was found in the violation of underlying assumption for the current oscillation method. When the amount of plasma produced (beginning and end of discharge pulse) is not constant, the positions of the ion current maxima and minima are not just determined by the drift velocities. Furthermore, the sets of data derived from the minima is not consistent with the data derived from maxima, which is do to the large asymmetry of the ion velocity distribution function. In principle, that effect could be use to estimate such asymmetry, 
however the large fluctuations ("noise") of the vacuum arc plasma does not justify such attempt. We conclude that the results of the current spike method are more reliable and therefore preferred.

2. The current spike method showed that

- The most likely ion velocity slightly decreases as the plasma flows away from the cathode spot region. This is very likely due to collisions of the ions with the "background" of neutrals. The presence and density evolution of neutrals was established in previous work.

- The ion velocity is slightly increased when the discharge current is increased. Such increase is correlated with an increase in the arc burning voltage and corresponding power dissipation in the nearcathode region.

- The plasma is faster when it is "born" in a non-uniform axial field and the anode is magnetically isolated. Also here, greater velocities correlate with higher arc burning voltage.

\section{Acknowledgments}

Two of us (S.H. and Ch. G.) thank Prof. J. Schein, UBW Munich, for arranging the research opportunity at Berkeley. This work was supported by the U.S. Department of Energy, Office of Nonproliferation and International Security, Initiatives for Proliferation Prevention, Project No. IPP-LBNLT2-196, under Contract No. DE-AC02-05CH11231 with the Lawrence Berkeley National Laboratory. 


\section{References}

[1] Tanberg R Phys. Rev. 193035 1080-1089.

[2] Plyutto A A, Ryzhkov V N, and Kapin A T Sov. Phys. JETP 196520 328-337.

[3] Davis W D and Miller H C J. Appl. Phys. 196940 2212-2221.

[4] Bilek M M M, Martin P J, and McKenzie D R J. Appl. Phys. 199883 2965-2970.

[5] Yushkov G Y, Anders A, Oks E M, and Brown I G J. Appl. Phys. 200088 5618-5622.

[6] Anders A and Yushkov G Y J. Appl. Phys. 200291 4824-4832.

[7] Tarrant R N, Bilek M M M, Oates T W H, Pigott J, and McKenzie D R Surf. Coat. Technol. 2002 $156110-114$.

[8] Byon E and Anders A J. Appl. Phys. 200393 1899-1906.

[9] Rosén J, Anders A, Mráz S, Atiser A, and Schneider J M J. Appl. Phys. 200699 123303-1-5.

[10] Rosén J, Schneider J M, and Anders A Appl. Phys. Lett. 200689 141502-1-3.

[11] Wieckert C Phys. Fluids 198730 1810-1813.

[12] Hantzsche E J. Phys. D: Appl. Phys. 199124 1339-1353.

[13] Hantzsche E IEEE Trans. Plasma Sci. 199523 893-898.

[14] Yushkov G, "Measurements of directed ion velocity in vacuum arc plasmas by arc current perturbation methods,” Proc. of XXth Int. Symp. on Discharges and Electrical Insulation in Vacuum, Xi'an, P.R. China, 2000, 260-263.

[15] Yushkov G Y, Oks E M, Anders A, and Brown I G J. Appl. Phys. 200087 8345-8350.

[16] Anders A, Oks E M, and Yushkov G Y J. Appl. Phys. 2007102043303.

[17] Chen F F, Plasma Physics and Controlled Fusion. New York: Plenum Press, 1984.

[18] Anders A and Yushkov G Y Appl. Phys. Lett. 200791091502. 


\section{Figure Captions}

Fig. 1 Experimental setup: the cathode rod (red) is placed inside an anode tube (blue) around which a coil is wound. The coil can be synchronously pulsed with the pulsed arc. The plasma is streaming to the left where a set of negatively biased ion collectors is positioned to recorded density variation propagating with the flowing plasma. The blue and black lines indicates the size and shape of anode and vacuum chamber components. The last (left) probe was $55 \mathrm{~cm}$ from the cathode front face.

Fig. 2 Example of oscillating arc and probe current, the latter is delayed relative to the arc current due to the time-of-flight of the plasma to the probe location;

Fig. 3 Arc voltage and arc current with the superimposed spike. The spike position can be selected relative to the arc initiation; the delay was here about $150 \mu \mathrm{s}$.

Fig. 4 Spatial and time-resolved evolution of the plasma flow velocity, tungsten plasma, no magnetic field. Detector 1 at $6 \mathrm{~cm}$ distance was not included here because detectors are off-axis and this one has no direct line-of-sight to the cathode.

Fig. 5 As Fig. 4 but for platinum.

Fig. 6 As Fig. 4 but for aluminum.

Fig. 7 Evolution of the plasma flow velocity for titanium plasma: (a) without external magnetic field, (b) with external field applied, having a maximum of $30 \mathrm{mT}$ in the center of the coil, and (c) with the maximum of the B-field $60 \mathrm{mT}$; the data points for (a)-(c) were obtained using the maxima of current and ion current curves (Fig. 2), whereas the results (d)-(e) where derived using the using the minima.

Fig. 8 Aluminum plasma velocity as a function of distance and time after triggering, as determined by the spike perturbation method.

Fig. 9 Ion velocity as a function of arc current, as determined with the spike perturbation method; aluminum plasma; no external magnetic field.

Fig. 10 Ion velocity as a function of external magnetic field, as determined by the spike perturbation method; aluminum plasma; arc current constant at $200 \mathrm{~A}$. 


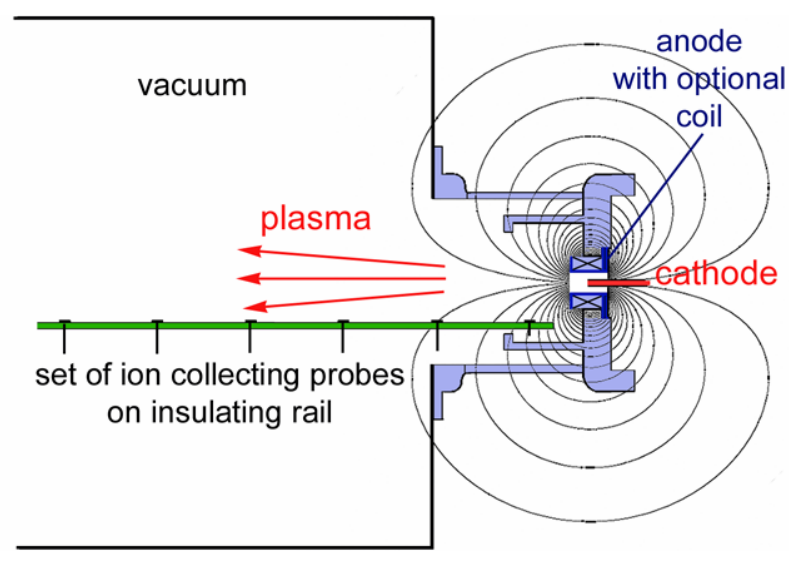

Fig. 1 


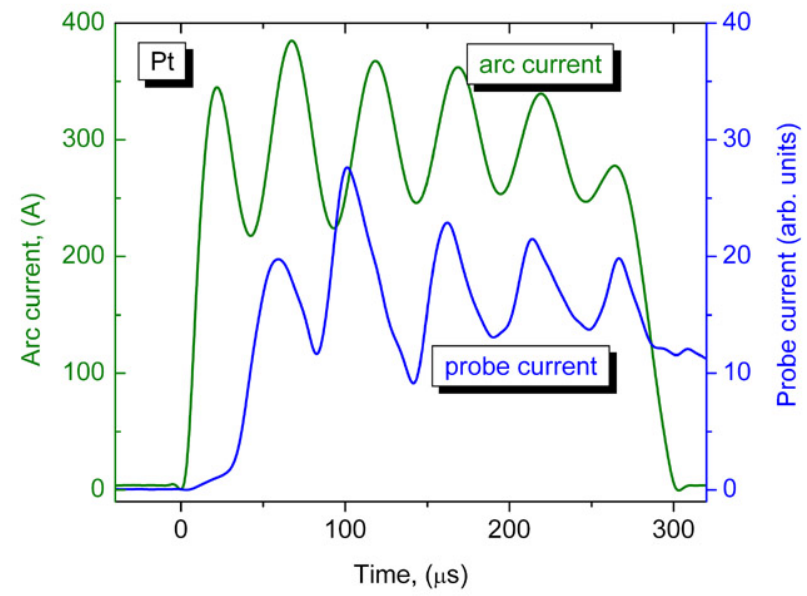

Fig. 2 


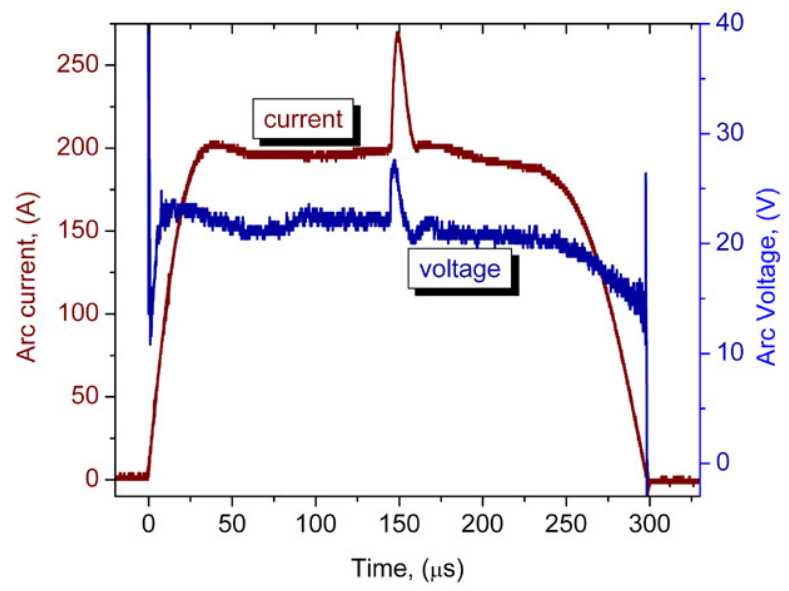

Fig. 3 


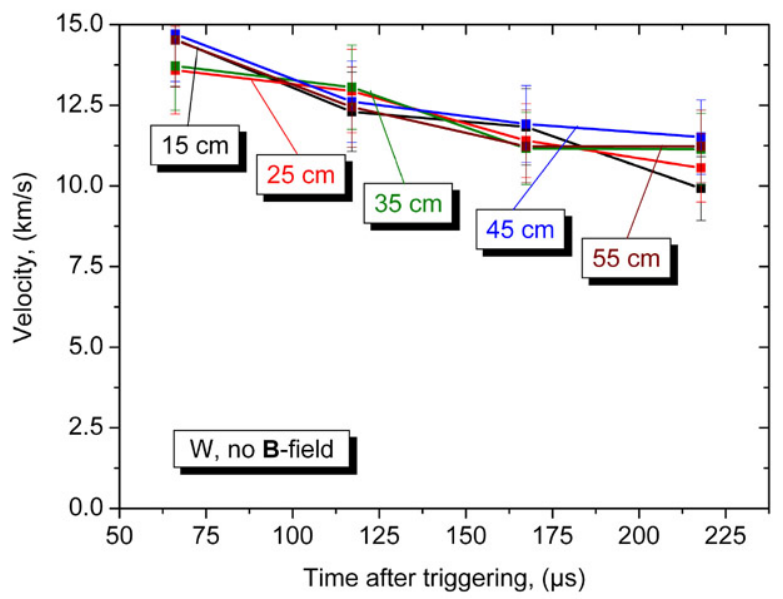

Fig. 4 


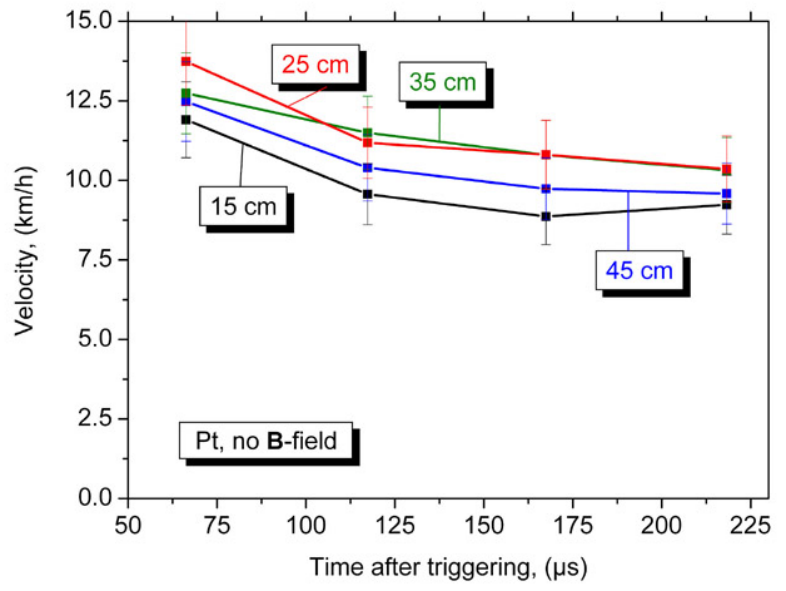

Fig. 5 


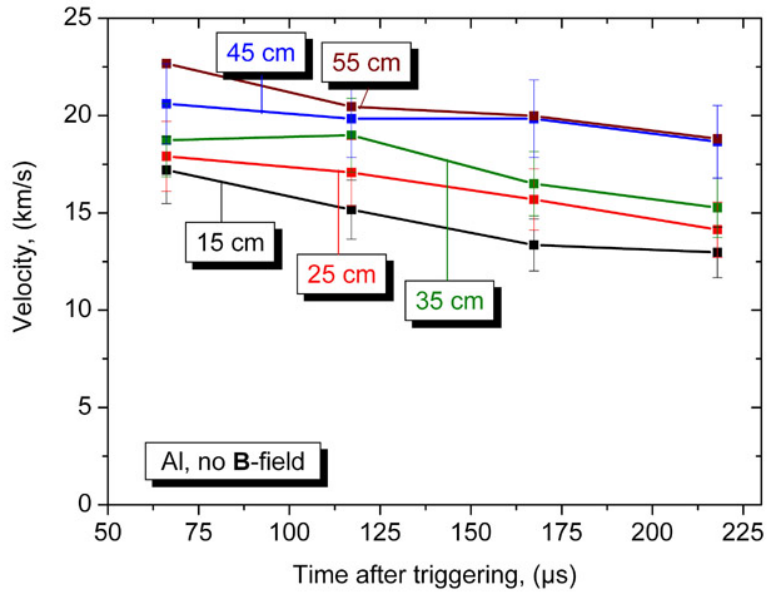

Fig. 6 

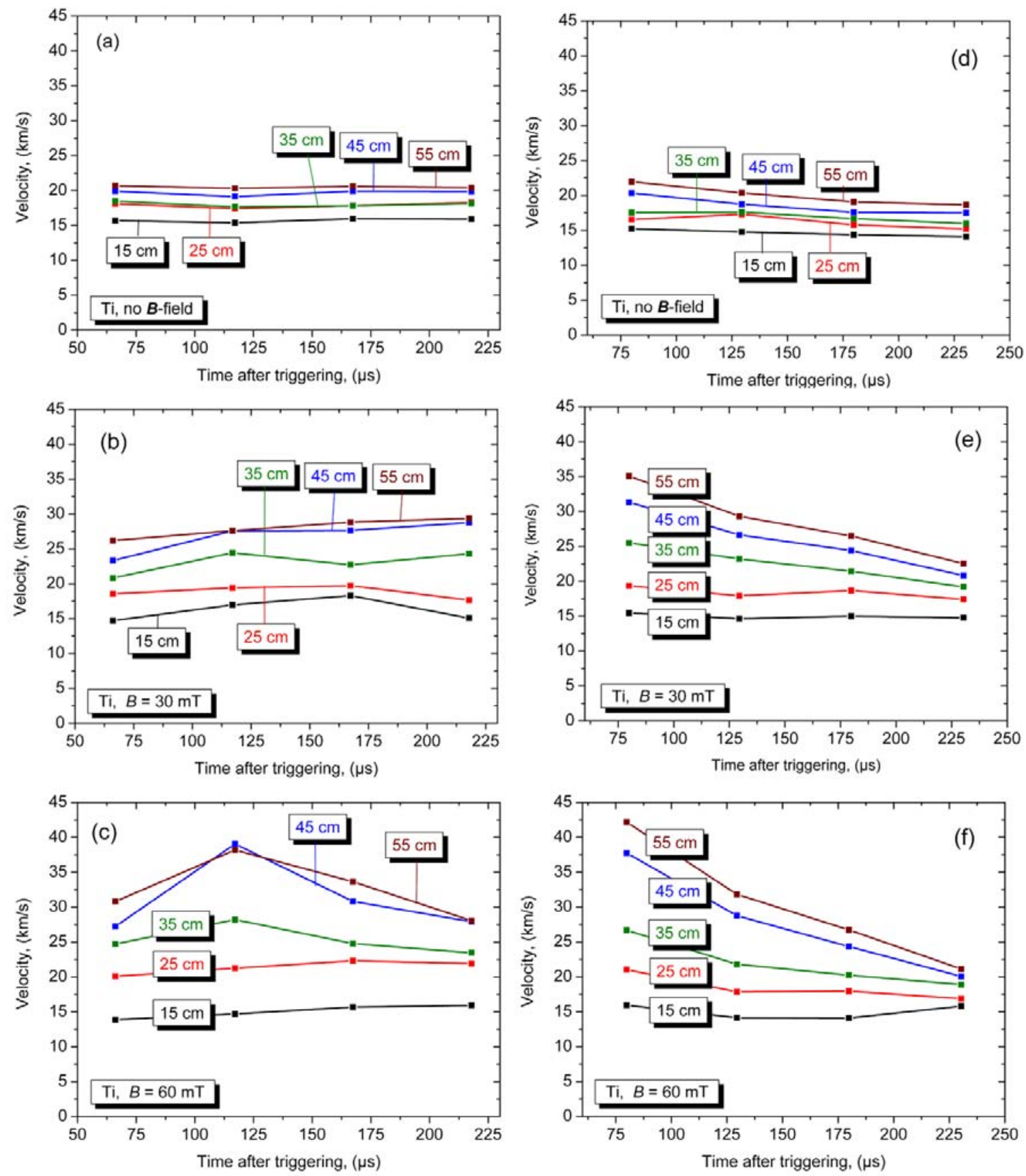

Fig. 7 


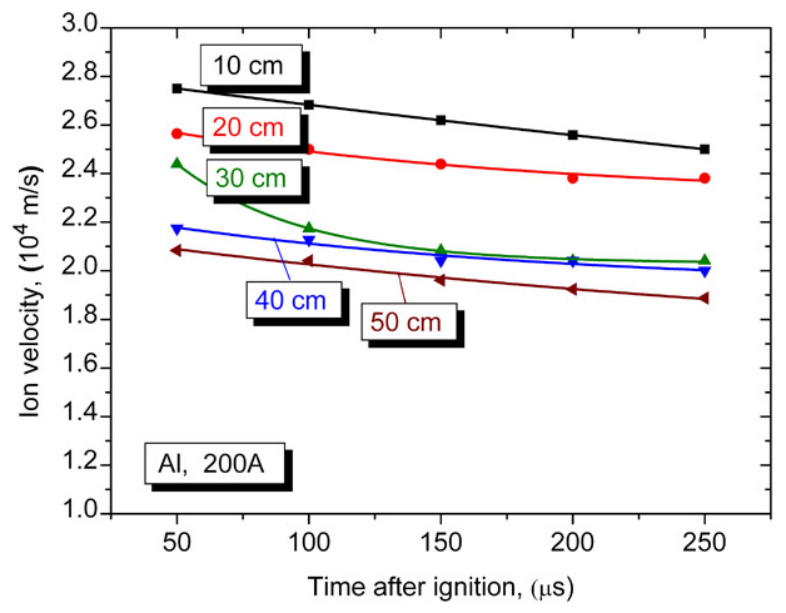

Fig. 8 


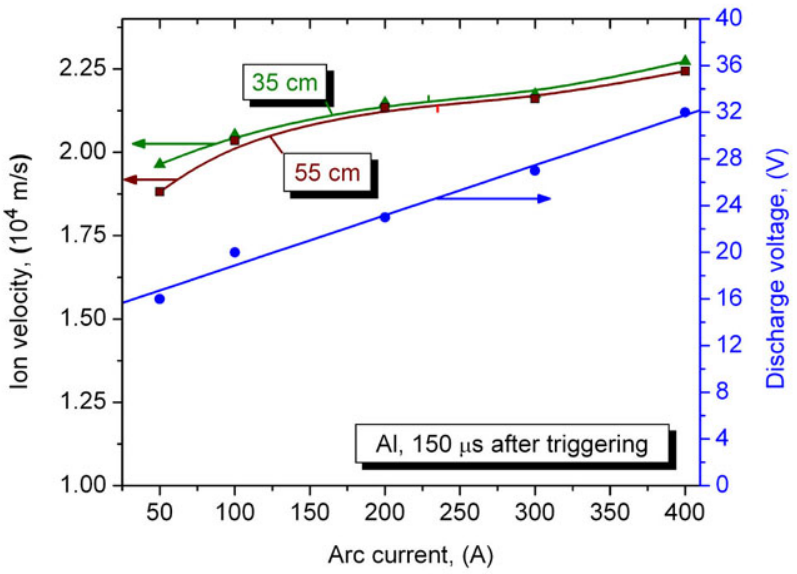

Fig. 9 


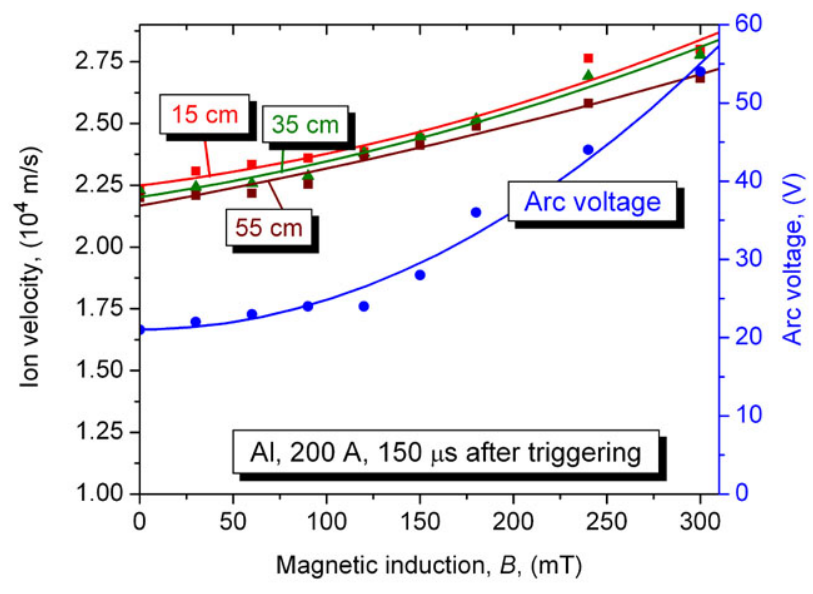

Fig. 10 